\title{
Purkinje cell COX deficiency and mtDNA depletion in an animal model of spinocerebellar ataxia type 1
}

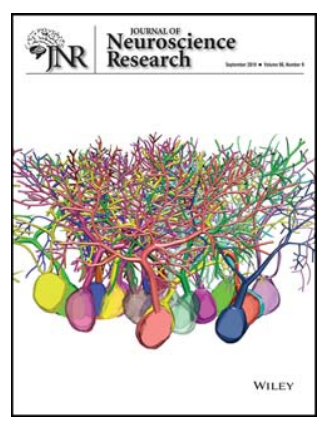

\author{
Michela Ripolone ${ }^{1}$ () | Valeria Lucchini ${ }^{1}$ | Dario Ronchi ${ }^{2}$ | \\ Gigliola Fagiolari $^{1}$ | Andreina Bordoni ${ }^{2}$ | Francesco Fortunato ${ }^{2}$ | \\ Stefania Mondello $^{3}$ | Sara Bonato ${ }^{2}$ | Mirella Meregalli ${ }^{4}$ | Yvan Torrente $^{4}$ | \\ Stefania Corti ${ }^{2}$ | Giacomo P. Comi ${ }^{2}$ | Maurizio Moggio ${ }^{1}$ | Monica Sciacco ${ }^{1}$
}

${ }^{1}$ Neuromuscular and Rare Diseases Unit, Department of Neuroscience, Fondazione IRCCS Ca' Granda, Ospedale Maggiore

Policlinico, Milan, Italy

${ }^{2}$ Neurology Unit, Neuroscience Section, Department of Pathophysiology and Transplantation, Dino Ferrari Centre, IRCCS Foundation Ca' Granda Ospedale Maggiore Policlinico, University of Milan, Milan, Italy

${ }^{3}$ Department of Biomedical and Dental Sciences and Morphofunctional Imaging, University of Messina, Messina, Italy

${ }^{4}$ Department of Pathophysiology and Transplantation, Stem Cell Laboratory, Università degli Studi di Milano, Fondazione IRCCS Ca' Granda Ospedale Maggiore Policlinico di Milano, Centro Dino Ferrari, Milan, Italy

\section{Correspondence}

Monica Sciacco, Neuromuscular and Rare Diseases Unit, Department of Neuroscience, Fondazione IRCCS Ca' Granda, Ospedale Maggiore Policlinico, via F. Sforza 35, 20122 Milan, Italy. Email: monica.sciacco@policlinico.mi.it

\section{Funding information}

Fondazione Cariplo Grant 2014-1010 (to Dario Ronchi and Associazione Amici del Centro Dino Ferrari-University of Milan)

\begin{abstract}
Spinocerebellar ataxias (SCAs) are a genetically heterogeneous group of cerebellar degenerative disorders, characterized by progressive gait unsteadiness, hand incoordination, and dysarthria. Ataxia type 1 (SCA1) is caused by the expansion of a CAG trinucleotide repeat in the SCA1 gene resulting in the atypical extension of a polyglutamine (polyQ) tract within the ataxin-1 protein. Our main objective was to investigate the mitochondrial oxidative metabolism in the cerebellum of transgenic SCA1 mice. SCA1 transgenic mice develop clinical features in the early life stages (around 5 weeks of age) presenting pathological cerebellar signs with concomitant progressive Purkinje neuron atrophy and relatively little cell loss; this evidence suggests that the SCA1 phenotype is not the result of cell death per se, but a possible effect of cellular dysfunction that occurs before neuronal demise. We studied the mitochondrial oxidative metabolism in cerebellar cells from both homozygous and heterozygous transgenic SCA1 mice, aged 2 and 6 months. Histochemical examination showed a cytochrome-c-oxidase (COX) deficiency in the Purkinje cells (PCs) of both heterozygous and homozygous mice, the oxidative defect being more prominent in older mice, in which the percentage of COX-deficient PC was up to $30 \%$. Using a laser-microdissector, we evaluated the mitochondrial DNA (mtDNA) content on selectively isolated COX-competent and COXdeficient PC by quantitative Polymerase Chain Reaction and we found mtDNA depletion in those with oxidative dysfunction. In conclusion, the selective oxidative metabolism defect observed in neuronal PC expressing mutant ataxin occurs as early as 8 weeks of age thus representing an early step in the PC degeneration process in SCA1 disease.
\end{abstract}

\section{KEYWORDS}

laser microdissector, mitochondria, mitochondrial DNA depletion, oxidative damage, Purkinje cell, spinocerebellar ataxia type 1 , transgenic mice

\section{1 | INTRODUCTION}

Spinocerebellar ataxia type 1 (SCA1) is an autosomal dominant inherited neurodegenerative disease characterized by neurodegeneration in multiple central nervous system (CNS) regions, including spinal cord, brain stem, and cerebellum (Zoghbi \& Orr, 1995).
The disease is associated with an unstable trinucleotide CAG repeat expansion in the open reading frame of the ataxin-1 gene. This specific CAG repeat expansion leads to the expression of an expanded polyglutamine (polyQ) tract in the mutant ataxin-1 protein (ATXN1), thereby acquiring a toxic gain-of-function property (Orr, 2012; Zoghbi, 1995; Zoghbi \& Orr, 2009). 


\section{Significance}

This work uses an animal model to try to understand the pathogenesis of a progressive human disease (spinocerebellar ataxia type 1, SCA1) characterized by progressive gait unsteadiness, hand incoordination, and dysarthria and transmitted as an autosomal dominant disorder. In particular, we focused our studies on the progressive dysfunction of Purkinje cells (PCs) and we hypothesize that a mitochondrial oxidative defect represents an early pathogenetic step of the degeneration process. Understanding the pathogenesis is extremely important because it can help the development of therapeutic strategies aiming at preserving PCs, delaying disease onset, and slowing its progression.

Also, it was showed that the overexpression of the normal form of ataxin-1, produces similar toxic effects (Fernandez-Funez et al., 2000), suggesting that an activity of ATXN1 in excess is sufficient to cause neuronal dysfunction.

Although genetic analyses, epidemiological data, neuropathological investigations are providing important new insights into the etiology of spinocerebellar ataxias (SCAs), the pathological mechanism of these diseases is still poorly understood (Duenas, Goold, \& Giunti, 2006), and the toxic mechanisms by which the ataxin-1 protein induces neuronal Purkinje cells (PCs) death has been investigated.

Different mouse models have been generated to study the cerebellar degeneration associated with SCA1 (Burright et al., 1995; Watase et al., 2002).

The transgenic animal model, generated by Burright et al. (1995) at the University of Minnesota, expresses the full length human SCA1 cDNA with 82 CAG repeats controlled by a specific Purkinje cell protein-2 (Pcp2) promoter (Oberdick, Smeyne, Mann, Zackson, \& Morgan, 1990; Vandaele et al., 1991).

This mouse model develops a progressive motor disorder starting in the early stages of life (around 5 weeks of age), before the appearance of any alteration of cerebellar morphology. Pathological abnormalities of PC, in the form of a shrunken dendritic tree and migration of the cell body into the molecular layer, become clearly visible by 12 weeks of age, along with an initial decline in PC number. A significant PC loss (approximately a third) occurs by 24 weeks of age. Interestingly, some authors suggested that neuronal dysfunction rather than neuronal loss is central to the disease process (Clark et al., 1997).

Mitochondrial dysfunction and oxidative damage are prominent features of various neurodegenerative diseases such as Amyotrophic Lateral Sclerosis, Spinal Muscular Atrophy, Alzheimer Disease, Parkinson's disease, Huntington Disease, hereditary spastic paraplegia, Friedreich's ataxia, and Charcot-Marie-Tooth disease (Allen, Duffy, Shaw, \& Grierson, 2015; DiMauro \& Schon, 2008; Ripolone et al., 2015).

Neurons are particularly sensitive to changes in mitochondrial function as they are extremely energy-dependent (Gironi et al., 2004; Kann \& Kovács, 2007). Being the apoptotic pathways related to mitochondrial dysfunction (Clark et al., 1997; Duenas et al., 2006), some evidences have shown that in some forms of cell damage occurring in polyglutaminediseases the observed phenotype may be related to apoptotic cell death.
Herein, searching for the possible explanation of cerebellar dysfunction, we tried to gain insight into the pathogenesis of this disease studying the mitochondrial oxidative metabolism in vivo in the cerebellum of transgenic SCA1 mice. In this view, considering the onset of the clinical symptoms, we decided to study mice at 2 months of age, soon after the beginning of symptoms, and then at the age of 6 months when both clinical symptoms and cerebellar alteration are clearly manifest.

We compared the histopathological changes, the presence of apoptosis, and the mitochondrial oxidative enzymatic activity in these mice. Also, using a laser-microdissector, we evaluated the mitochondrial DNA (mtDNA) content on selectively isolated COX-competent and COX-deficient PC by quantitative Polymerase Chain Reaction (PCR).

\section{2 | MATERIALS AND METHODS}

\section{$2.1 \mid$ Animals}

All experiments in this study were performed on SCA1 transgenic mice and control mice generated by Dr. H. Orr at the University of Minnesota (Burright et al., 1995) and kindly supplied by Dr. Pandolfo, Université Libre de Bruxelles, Bruxelles (Belgium). A colony of heterozygous and homozygous SCA1 B05 82Q mice was kept in our animal facility, animals being maintained at $22^{\circ} \mathrm{C} \pm 1^{\circ} \mathrm{C}$ on a 12 hr light/12 hr dark schedule, with free access to food and water. All protocols for animal care, handling, and euthanasia were performed in accordance with the guidelines for the care and use of Laboratory Animals promulgated by the Italian Minister of Health, DL 27 January 1992, No. 116 and the EU directive 86/609/CEE. Mice were sacrificed with cervical dislocation, through occipital access brain was taken up to brainstem. It was frozen with quick immersion in liquid nitrogen-cooled isopentane and then in liquid nitrogen. Frozen tissues were mounted on the ventral side and cut in cross sections. Thirty-six mice (twenty males and sixteen females) were sacrificed and studied, namely six heterozygous and six homozygous SCA1 transgenic 2-month old mice, six heterozygous and six homozygous SCA1 transgenic 6-month old mice, and twelve control mice (six 2 months old, six 6 months old).

\section{2 | Histopathological and biochemical analysis}

Histological staining and histochemical reactions both for detection of morphological CNS alterations and mitochondrial enzymatic defects were performed on 8- $\mu \mathrm{m}$ thick transverse cryostat sections from mouse whole brains prepared as described (Ripolone et al., 2015; Sciacco \& Bonilla, 1996).

Morphological evidence for apoptosis was tested using terminal deoxynucleotidyl transferase-mediated dUTP nick end labeling (TUNEL). The TUNEL method was set up following the manufacturer's instructions (In Situ Cell Death Detection Kit, Roche, Mannheim, Germany) and according to our previous studies (Sciacco et al., 2001). 
TUNEL sections were compared with serial methylene blue stained sections.

For immunohistochemistry, cryostatic cerebellum sections $(8 \mu \mathrm{m}$ thick) were postfixed in $4 \%$ Paraformaldehyde (Sigma-Aldrich, S.r.l. Milan, Italy), blocked in 3\% Bovine Serum Albumin (Sigma-Aldrich), and incubated with primary and secondary antibodies at the appropriate dilution. Primary antibodies were anti-calbindin D-28K polyclonal antibody (diluition: 1:500, 2d-Chemicon, Thermo Fisher Scientific, Milan, Italy), anti-OxPhos Complex IV subunit I monoclonal antibody (diluition 1:500, ON Invitrogen), anti-TOMM20 monoclonal antibody (diluition 1:250 ON Abcam, Cambridge, United Kingdom). Alexa 488- or Alexa 568-conjugated IgGs were used as secondary antibodies (Invitrogen, Carlsbad, CA).

Biochemical analyses were performed on the $750 \mathrm{~g}$ supernatants obtained from brains and cerebellum homogenates. Enzyme activities of respiratory chain complexes I, II, III, IV (cytochrome-coxidase, COX) and of citrate synthase (CS) were measured spectrophotometrically and the specific activities of each complex were normalized to CS, an indicator of the number of mitochondria (Bresolin et al., 1987).

\subsection{Electron microscopy}

For ultrastructural examination, a small part of cerebellum samples was fixed in $2.5 \%$ glutaraldehyde $(\mathrm{pH} 7.4)$, postfixed in $2 \%$ osmium tetroxide and then, after dehydration in a graded series of ethanol, embedded in Spurr's resin. Finally, ultrathin sections were stained with lead citrate and uranyl acetate and examinated with Zeiss EM109 transmission electron microscope (Ripolone et al., 2015).

For immunoultrastructural evidence of COX enzymatic activity, small samples of cerebellum from 2- and 6-month old mice were incubated in 0.1M phosphate buffer, $\mathrm{pH} 7.4,5 \mathrm{mM} 3,3^{\prime}$-Diaminobenzidine tetrahydrochloride, $0.25 \mathrm{M}$ cytochrome $\mathrm{c}, 0.2 \mathrm{M}$ sucrose, for $1 \mathrm{hr}$ at $37^{\circ} \mathrm{C}$. After two washes in $0.1 \mathrm{M}$ phosphate buffer, $\mathrm{pH} 7.4$, samples were fixed in $2.5 \%$ glutaraldehyde $(\mathrm{pH} 7.4)$ and processed according to the standard protocol, ultrathin sections were not stained with lead citrate and uranyl acetate before the examination (Bonilla, Schotland, DiMauro, \& Aldover, 1975).

\subsection{Quantitative analysis of COX deficit in PCs}

Morphometric evaluation of COX-deficient and COX-competent PC was made on $12-\mu \mathrm{m}$-thick transverse cerebellar sections. Each section was entirely acquired at $100 \mathrm{X}$ with a Leica microscope equipped with a video-camera (Leica DCF 320) by using dedicated imaging software. Pictures were analyzed using the image analyzer software IM50 (Leica Image Manager IM50). Three different blinded operators counted COX-deficient and COX-positive PC in each picture following the protocol by Peverelli et al. (2015). We analyzed five non-transgenic (nonTG) samples, five heterozygous 2- and 6-month old mice (totally 10 samples) and five homozygous 2- and 6-month old mice (totally 10 samples).

\section{5 | Laser-capture microdissection and mtDNA analysis}

Frozen cryostat sections (16 $\mu \mathrm{m})$ were collected on 2-mm thick pen membrane slides, stained for COX activity detection, and air-dried. Individual COX-competent and -deficient PC were microdissected by using a Leica Laser Microdissection System (Leica AS-LMD) (Edwards, 2007).

PC from four 6-month old SCA1 homozygous and three control mice were dissected from each section and collected in groups of 300 cells per type into single test tubes. We also collected granular cells (GCs) from all dissected mice as internal control for both oxidative damage and mtDNA content.

DNA was extracted by laser-microdissected cells using the QIAamp DNA Micro Kit (QIAGEN, Italia, Milan, Italy).

Mouse mtDNA was quantified by Real time PCR using an $A B I$ 7500 detection system as previously described (Spinazzola et al., 2006). The primers and probe for murine mt-Co1, mt-Co3, mt-Nd4 genes (mtDNA), and for Tfrc and $18 \mathrm{~S}$ ribosomal RNA gene (nDNA) were used for that purpose and are available upon request. All the determinations were carried out in triplicate using $5 \mu$ l of cell lysates as a template. Nuclear-DNA normalized mtDNA levels were expressed in terms of relative quantification (RQ) values considering as reference $(R Q=1)$ the amount of mtDNA detected in PC from a control mouse using the Double Delta Cycle Threshold (Ct) Value ( $\Delta \Delta \mathrm{Ct})$ method. Briefly, mtDNA levels are obtained by applying this formula: 2 [(Ct $\left.t_{\text {mtDNATARGET_SCA1 }}-\mathrm{Ct}_{\text {nDNATARGET_sCA1 }}\right)-\left(\mathrm{Ct}_{\text {mtDNATARGET_CTRL }}-\right.$ $\left.\mathrm{Ct}_{\text {nDNATARGET_CTRL }}\right)$.

Analysis of mtDNA point mutation is described in Supporting Information.

\section{6 | Statistical analyses}

Data for all parameters measured were expressed as group means \pm standard deviation. There was no evidence for significant deviations from normal distribution ( $p>.05$, Shapiro-Wilk test). Group data were analyzed using one-way analysis of variance (ANOVA) followed by post hoc Tukey tests for multiple comparisons. Comparisons of mtDNA levels in GCs, brain, and cerebellum tissues between SCA1 and control mice were made using an unpaired $t$ test (with Bonferroni correction for multiple comparisons, as appropriate). The level of significance was set at $p<.05$. Statistical analyses were conducted using Prism 5.0 (GraphPad Sofware, Inc.) and STATA version 13.0 (StataCorp, College Station, TX).

\section{3 | RESULTS}

\subsection{PC mitochondrial oxidative defect in SCA1 mice, histochemical and ultrastructural examination}

To investigate the mitochondrial defect in SCA1 mice, we investigated histological examination and histochemical reactions for COX on serial cryostat sections on both homozygous and heterozygous 2 and 6 months old mice, and compared with those from control animals (Figure 1a-h). 

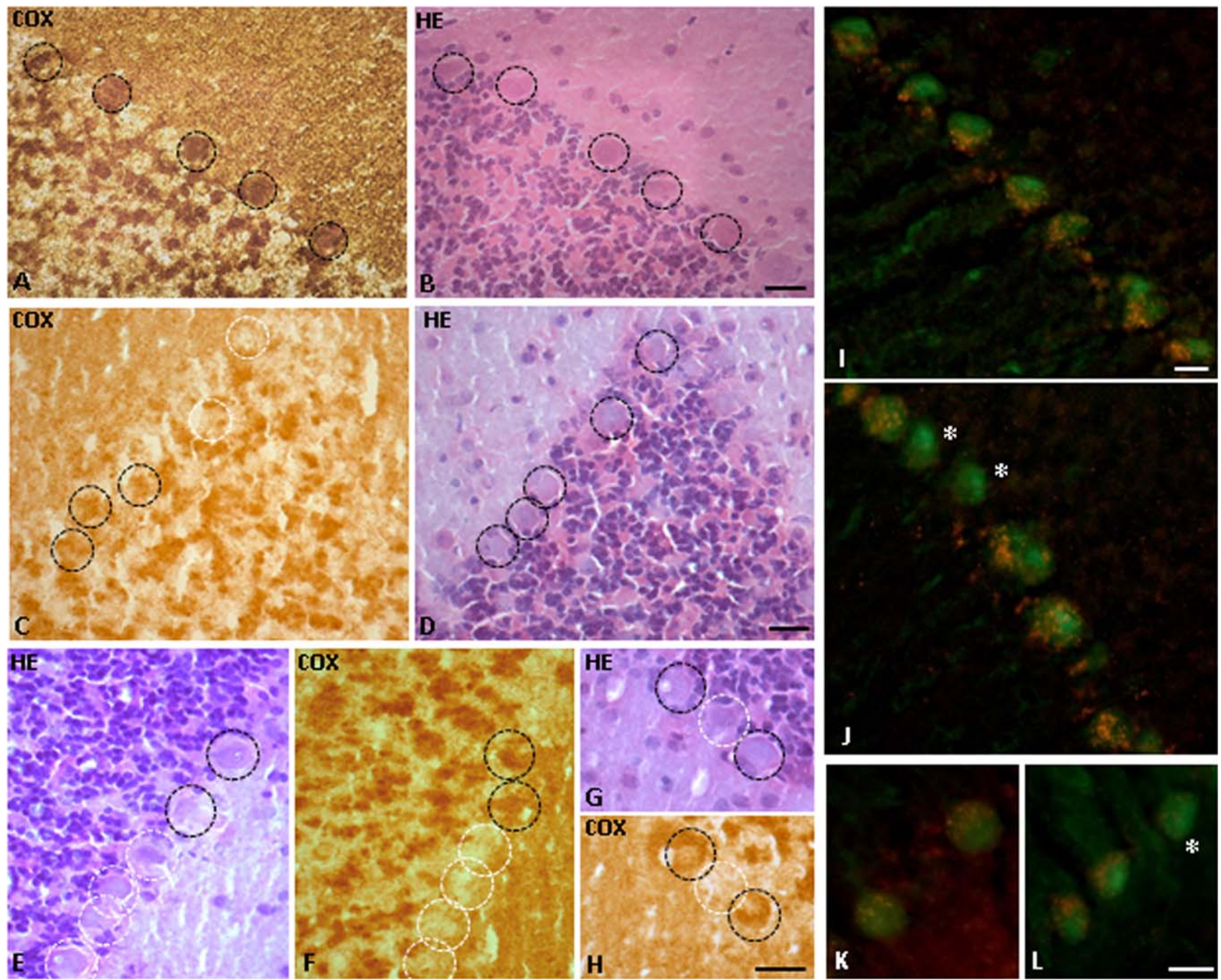

FIGURE 1 Light microscopy: histology-histochemistry-immunohistochemistry. Serial sections showing cerebellum from a non-TG mouse (a, b), a 2-month old spinocerebellar ataxia type 1 (SCA1)-homozygous mouse (c, d), and a 6-month old SCA1-homozygous mouse (e-h). The type of staining is indicated on each photo. Cytochrome-c-oxidase (COX)-positive and COX-negative cells are indicated by black and white circles, respectively. Co-immunostaining with both anti-COX1 subunit and anti-calbindin antibodies is shown on Purkinje neurons from a non-TG mouse ( $\mathrm{i}, \mathrm{k}$ ) and from a 6-month old SCA1-homozygous mouse (j, I). COX-deficient neurons are marked with asterisks. Scale bar: $15 \mu \mathrm{m}$

General morphological analyses were in line with previous data (Burright et al., 1995; Clark et al., 1997) indeed SCA1 transgenic mice present clinical features of cerebellar dysfunction.

In detail, we observed a mild loss of the PC population in the cerebellar cortex of both heterozygous and homozygous 2 months old mice. The loss of PC became more evident in both heterozygous and homozygous 6 months old mice, in which we observed some PC in heterotopic positions within the molecular layer and, occasionally, in the granular layer.

To evaluate the potential role of apoptosis in the degeneration of PCs, we performed Tunel experiments, but we found apoptosis in very few PC in both heterozygous and homozygous 2 and 6 months old mice. We therefore conclude that apoptosis does not seem to be involved in PC degeneration (Supporting Information Figure 1).

The histochemical examination showed similar amounts of COXdeficient PC in both heterozygous and homozygous mice, although, the oxidative defect was more pronounced in older (6 months old) mice (Figure 1).

To precisely assess the morphologically observed oxidative defect, we performed a quantitative analysis of the COX-deficient and COX-competent PC number. In addition to correctly recognize COX deficiency in PC, we performed co-immunohistochemistry of the respiratory chain Complex IV (subunit I) and calbindin, a marker of Purkinje neurons, by using appropriate antibodies (Figure 1i-I). We found that the percentage of COX-deficient PC over the total number of $\mathrm{PC}$ was significantly different across experimental conditions $\left(F_{4,20}=65.45, p<.001\right)$. In particular, it was substantially increased in both homozygous and heterozygous animals aged 2 and 6 months compared with controls $(\sim 12 \%, p<.001)$. Furthermore, a higher number of COX-deficient PC was observed in 6month old mice compared with younger ones $(\sim 30 \%, p<.001)$ (Supporting Information Figure 2). 

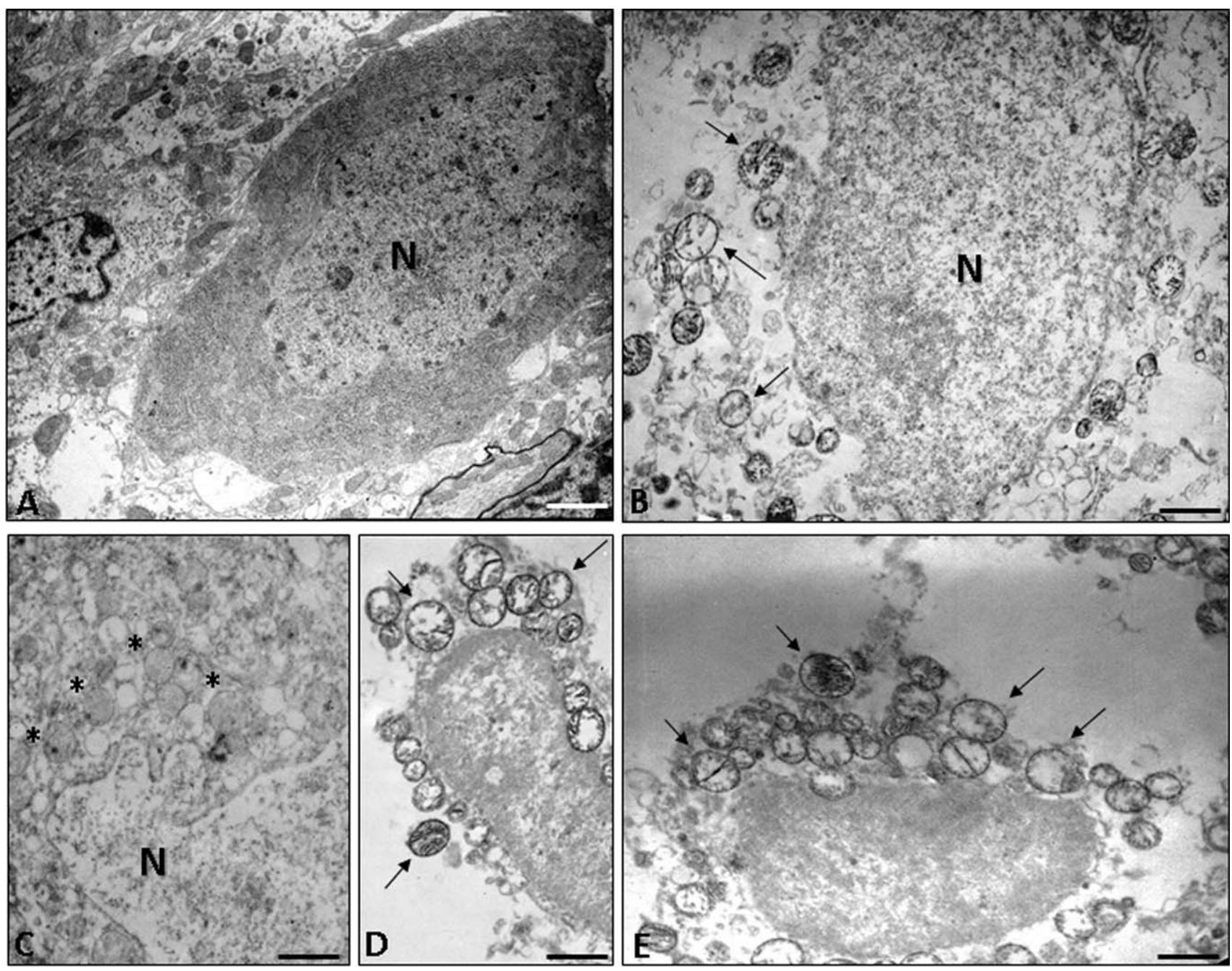

FIGURE 2 Electron microscopy: cytochrome-c-oxidase (COX) immunostaining in 6 months old control and spinocerebellar ataxia type 1 (SCA1)-homozygous mice. (a) Purkinje cell (PC) from a non-TG mouse without COX immunostaining. Scale bar: $2200 \mathrm{~nm}$. (b) COX enzymatic activity in non-TG PC. All mitochondria show COX activity (arrows). Scale bar: $1400 \mathrm{~nm}$. (c) COX enzymatic activity in a PC from SCA1homozygous 6 months old mouse. COX-negative mitochondria are marked with an asterisk. Scale bar: 1600 nm. (d) COX enzymatic activity in a PC from SCA1-homozygous 6 months old mouse. COX-positive mitochondria are marked with arrows. Scale bar: $1400 \mathrm{~nm}$. (e) COX enzymatic activity in SCA1-homozygous granular cell. All mitochondria show COX activity (arrows). Scale bar: $1400 \mathrm{~nm}$. $\mathrm{N}=\mathrm{nucleus}$

Finally, ultrastructural immunostaining for COX activity confirmed that the lack of enzymatic activity was confined to PC as shown in Figure 2. Indeed, many mitochondria membranes were not electron dense in SCA1 mice, while they are clearly electron dense in both controls and in granular cerebellar cells from SCA mice (Figure 2).

Taken together, our experimental evidences show a selective COX deficiency in a significant amount of PC from genetically affected mice.

\section{2 | Biochemical analysis}

Enzymatic assay of respiratory chain activities was performed on cerebellar tissue of five heterozygous and five homozygous SCA1 transgenic mice aged 6 months and in five controls. The values are presented in Table 1. The activities of cll + III and cIV complexes, as well as the activity of CS, in both groups of SCA1 transgenic mice differed significantly from controls (Table 1).
There was a significant increase in cll/CS activity in heterozygous animals compared with controls. cll/CS activity was also increased in homozygous mice but not significantly. A trend toward a higher $\mathrm{cl}+\mathrm{III}$ activity in transgenic mice was observed. No significant differences were found between SCA1 heterozygous and homozygous mice (Table 1).

\section{3 mtDNA molecular analysis in SCA1 mice cerebellum}

\subsection{1 | mtDNA point mutation analysis}

Sequencing analysis revealed less than one point mutations per $10 \mathrm{kbp}$ in CytB region of mtDNA amplified from COX-positive and COXdeficient PC. No mutations were detected in clones representative of MT-COI and D-loop regions. 
TABLE 1 Mean (standard deviation) for respiratory chain activities in heterozygous and homozygous spinocerebellar ataxia type 1 transgenic mice aged 6 months and in controls

\begin{tabular}{|c|c|c|c|c|c|c|}
\hline & \multirow{2}{*}{$\begin{array}{l}\text { Non-TG } \\
(n=5)\end{array}$} & \multirow{2}{*}{$\begin{array}{l}\text { Hetero } 6 \mathrm{~m} \\
(n=5)\end{array}$} & \multirow{2}{*}{$\begin{array}{l}\text { Homo } 6 \mathrm{~m} \\
(n=5)\end{array}$} & \multirow[b]{2}{*}{$p$ Value $^{a}$} & \multicolumn{2}{|l|}{$p$ Value $^{a}$ post hoc test } \\
\hline & & & & & Hetero $6 \mathrm{~m}$ vs. Non-TG & Homo $6 \mathrm{~m}$ vs. Non-TG \\
\hline $\mathrm{cl} / \mathrm{CS}$ & $10.22(0.77)$ & $8.73(1.37)$ & $10.51(1.57)$ & $\begin{array}{l}F_{2,12}=2.78 \\
p=.102\end{array}$ & ns & ns \\
\hline cll/CS & $2.07(0.48)$ & $3.27(0.312)^{* *}$ & $2.60(0.48)$ & $\begin{array}{l}F_{2,12}=9.66 \\
p=.003\end{array}$ & .002 & ns \\
\hline $\mathrm{Cl}+\mathrm{III} / \mathrm{CS}$ & $5.95(0.69)$ & $8.00(1.52)$ & $8.21(1.68)$ & $\begin{array}{l}F_{2,12}=4.17 \\
p=.042\end{array}$ & ns & ns \\
\hline $\mathrm{Cll}+\mathrm{III} / \mathrm{CS}$ & $1.22(0.15)$ & $2.21(0.41)^{* *}$ & $2.73(0.56)^{* * *}$ & $\begin{array}{l}F_{2,12}=17.51 \\
p<.001\end{array}$ & .006 & $<.001$ \\
\hline $\mathrm{clV} / \mathrm{CS}$ & $14.70(2.65)$ & $31.01(8.40)^{* *}$ & $36.16(5.93)^{* * *}$ & $\begin{array}{l}F_{2,12}=16.70 \\
p<.001\end{array}$ & .003 & $<.001$ \\
\hline CS & $435.40(23.21)$ & $358.30(46.52)^{* *}$ & $330.20(22.21)^{* * *}$ & $\begin{array}{l}F_{2,12}=13.92 \\
p<.001\end{array}$ & .007 & $<.001$ \\
\hline
\end{tabular}

Note. $\mathrm{ns}=$ not significant; Non-TG $=$ non-transgenic. Values are expressed as mean \pm standard deviations $(\mathrm{pmol} / \mathrm{min} / \mathrm{mg})$, after normalization to citrate synthase (CS). CS activity is normalized to protein content.

The bold values are statistically significant.

${ }^{a}$ One-way analysis of variance with Tukey's post hoc test.

${ }^{* *} p<.01 ;{ }^{* * *} p<.001$ versus controls.

\subsection{2 | mtDNA depletion}

mtDNA content was assessed in laser-microdissected PC by quantitative PCR determinations in a series of RQ experiments. Levels of mtDNA in COX-deficient PC from SCA1 mice were significantly decreased compared with COX-competent PC isolated from SCA1 and control mice (Figure 3; Supporting Information Table 1). mtDNA content was not always homogenous in the analyzed mice. Nevertheless, the ratio of mtDNA levels of isolated COX-deficient and COX-positive PC showed a strong mtDNA depletion ranging from 54 to $89 \%$ in all the mutant mice. Notably, these findings were limited to PC, as we detected no difference in mtDNA content of GCs isolated from SCA1 and control mice $(p>.05)$. We also quantified mtDNA in whole brain and cerebellum tissues. Levels of brain-derived mtDNA were not significantly different between SCA1 and control mice $(p>.05)$. Conversely, mtDNA levels were significantly reduced in cerebella of SCA1 mice (Supporting Information Figure 3). We suggest that the observed low mtDNA content in PC of SCA1 mice is not a result of the reduction of the amount of mitochondria, indeed immunofluorescence analysis of the outer mitochondrial membrane protein TOMM20, a marker of mitochondrial content, has a similar pattern of intensity in SCA1 and in control mice (Supporting Information Figure 4C,D).

Also, standard electron microscopy examination confirmed the presence of a normal amount of normally shaped and distributed PC mitochondria in the cerebellum of both transgenic and control mice (Supporting Information Figure 4A,B).

\section{4 | DISCUSSION}

SCA1 is a late-onset autosomal dominant neurodegenerative disorder of the nervous system caused by the toxic effects triggered by an expanded polyglutamine within ataxin-1 protein. The neuronal degeneration afflicts primarily the cerebellar PC and brainstem neurons, leading to the characteristic ataxic phenotype and bulbar dysfunction.

In SCA1 transgenic mice, the overexpression of mutant ataxin-1 results in a progressive ataxia and $\mathrm{PC}$ pathology is very similar to those seen in SCA1 patients (Burright et al., 1995).

Interestingly, the pathology develops as a result of an early neuronal dysfunction and atrophy, without neuronal loss (Clark et al., 1997), making the model helpful to define the causes of PC dysfunction and to study the disease pathogenesis.

Our main objective was to investigate the mitochondrial oxidative activity in cerebellar cells of transgenic SCA1 mice trying to correlate the dysfunction with the earliest histopathological changes and possible mtDNA defects.

In this regard, we studied both heterozygous and homozygous SCA1 transgenic mice, aged 2 and 6 months, by histological and histochemical techniques.

Our results showed a selective COX deficiency in several PC from pathologic mice, COX activity being normal in all other cerebellar cells and in control mice.

In particular, we detected similar amounts of COX-deficient PC in both heterozygous and homozygous affected mice, the defect being more evident in the older ones, in which the percentage of COXdeficient PC was up to 30\% (Figure 1; Supporting Information Figure 1). The fact that heterozygous and homozygous transgenic mice express the oxidative defect at the same level indicates that the level of accumulation of the ataxin-1 protein in heterozygous mice is enough to determine the neuronal damage.

Similarly, the other SCA1 mouse model, SCA1 154Q/2Q (Watase et al., 2002) and the transgenic mice for AFG3L2, the gene responsible for autosomal dominant SCA28 (Di Bella et al., 2010), display cerebellar 


\section{mtDNA/nDNA (RQ)}
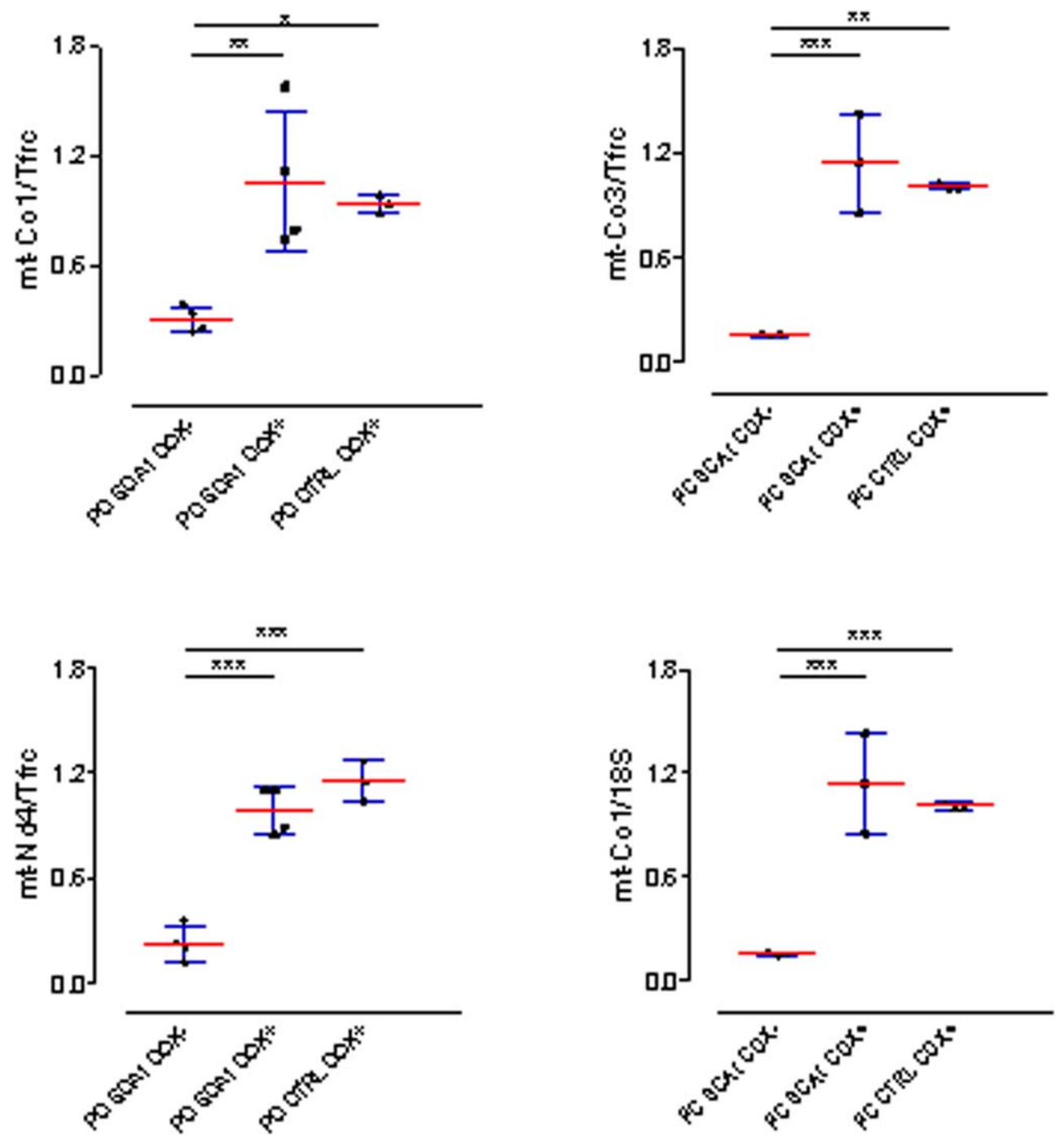

FIGURE 3 Quantitative mitochondrial DNA (mtDNA) analysis: mtDNA content in cytochrome-c-oxidase (COX)-deficient Purkinje cell (PC) and COX-competent PC isolated from spinocerebellar ataxia type 1 (SCA1) mice $(n=4)$ and control mice $(n=3)$. Cells were microdissected using a Leica Laser Microdissection System (Leica AS-LMD) from frozen cryostat sections $(16 \mu \mathrm{m})$ and collected in groups of 300 cells per type into single test tubes. Nuclear-DNA normalized mtDNA levels were expressed in terms of relative quantification (RQ) values considering as reference $(R Q=1)$ the amount of mtDNA detected in PC from controls. The primers and probe for murine $\mathrm{mt}-\mathrm{Co} 1, \mathrm{mt}-\mathrm{Co} 3, \mathrm{mt}-\mathrm{Nd} 4$ genes (mtDNA) and for Tfrc and 18S ribosomal RNA gene (nDNA) were used. The red horizontal line represents the mean and the error bars represent the standard deviation. Significant differences are indicated. ${ }^{*} p<.05 ;^{* *} p<.01 ;{ }^{* * *} p<.001$ (See Supporting Information Table 1 for details)

mitochondrial dysfunction at histochemical and molecular level (Maltecca et al., 2009; Stucki et al., 2016).

We also investigated the apoptotic phenomenon in PC degeneration, indeed, as apoptosis is involved in response regulation to cellular damage, it has been hypothesized that polyglutamine proteins activate the apoptotic machinery and that the cell loss is a central component of the disease (Paulson, Bonini, \& Roth, 2000). The apoptotic pathway had already been studied in SCA1 transgenic mice aged 15 and 20 weeks, and the authors had not found a significant number of TUNEL-positive PC (Shahbazian, Orr, \& Zoghbi, 2001). In agreement with these results, we only found few apoptotic nuclei in cerebellum cells which prevented us from performing a quantitative analysis (Supporting Information Figure 1).

The determination of COX activity by biochemical assay on homogenates of the whole cerebellum did not reveal any defect in the late disease stage, at 6 months of age. This result is largely explained by the different nature of the methods used to evaluate COX activity. The histochemistry allows to detect specific cell defects (affecting in this case a subset of PC only), whereas the biochemical assay pools in the same homogenate proteins of the entire cerebellum, therefore masking a specific cell defect (DiMauro et al., 2006). Complex IV (COX) 
activity was also preserved at an earlier time point ( 2 months age). However, in younger transgenic mice, we were able to detect a mild reduction cll + III activity as previously observed in presymptomatic SCA1-mice (Sánchez, Balagué, \& Matilla-Dueñas, 2016).

To search for an explanation of the observed selective oxidative defect, we studied the mtDNA content in both COX-deficient and COX-competent PC from 6 months old homozygous and control mice and in GC derived from SCA1 mice. To this purpose, we isolated and collected single, histochemically identified COX-positive/competent and COX-deficient PC, using a laser microdissector. After excluding an increased rate of point mutations in mtDNA, we observed a global reduction of mtDNA content in PC lacking COX activity while COXcompetent PC and GC were spared. We suggest that the observed low mtDNA content in PC of SCA1 mice is not a result of the reduction of the amount of mitochondria by comparing SCA1 and control mice in both ultrastructural images and TOMM20 intensity (Supporting Information Figure 4).

This distribution resembles the coexistence of COX-positive and cOX-deficient fibers found in skeletal muscle tissue from individuals affected with genetically determined mitochondrial disorders in which even a little percentage of COX deficient fibers can lead to muscle dysfunction (Barca et al., 2016; Horvath et al., 2012). mtDNA maintenance requires a pool of nuclear-encoded proteins whose dysfunction has been associated with several human diseases. Notably, autosomal recessive mutations in genes encoding the mtDNA polymerase POLG and the mitochondrial helicase Twinkle result in two ataxic syndromes, namely MIRAS (Winterthun et al., 2005) and infantile-onset SCA IOSCA (Nikali et al., 2005), respectively. Mutated patients only show minimal amount of mtDNA deletions and lack of mitochondrial myopathy, suggesting that defects in ubiquitous proteins of mtDNA replicative machinery can produce CNS-restricted degeneration.

Recently, Sánchez et al. (2016) also quantified the levels of mtDNA in cerebellum of SCA1 mice, but they found no significant change in mtDNA copy levels compared with control mice. Their experiments, however, were performed on 5 weeks old mice, that is, in a very early disease stage, and this may account for the divergence from our results.

The selective loss of specific neuronal subtypes is a central issue in neurodegenerative disorders.

As PC dendrites branch more extensively than those of any other neuron in the brain, they are probably more sensitive to reduced mitochondrial function. Gene expression analysis of mouse brain regions showed high expression levels of genes involved in mitochondrial ATP production (particularly those encoding COX subunits) in developing cerebellum, when the majority of granule cells are engaged in axon extension and synapse formation, and at a later stage (week 6) when cerebellum has reached maturity (Matoba et al., 2000). More recently Hosp et al. (2015) investigated the repertoire of proteins sequestered by mutant ataxin-1 in the SCA1-82Q mouse model while the proteome of PC cells in the Sca1(154Q/2Q) mice at a symptomatic stage was assessed by mass spectrometry (Stucki et al., 2016). Although no obvious factor involved in mtDNA maintenance were detected in these datasets, prominent alterations in mitochondrial proteins were found. Interestingly, mitochondrial morphology, found altered in affected PC cells, relies upon a set of proteins, which are also involved in mtDNA homeostasis (i.e., MFN2, OPA1, CHCHD10). It is noteworthy that mutations in these proteins are also associated with neurodegenerative disorders in human and models. For example, mitochondrial dysfunction has been previously reported in mouse PC lacking Mitofusin-2 (Mfn2), a factor involved in mitochondrial fusion pathway whose impairment is associated in humans with an autosomal dominant form of Charcot-Marie-Tooth disease (CMT2A) (Chen \& Chan, 2009). The authors documented the loss of mtDNA nucleoids in fusion-deficient cells as the likely basis of reduced respiratory chain function. Interestingly, loss of activity in electron transport chain complexes I and IV seems to precede neuronal death.

Finally, Ito et al. (2015) demonstrated that the impairment of mtDNA quality control due to loss of high-mobility group box 1 (HMGB1) protein is associated with the exacerbation of symptoms in the Atxn1-154Q knock-in mice.

Although inherited ataxias are extremely heterogeneous, caused by a variety of genes and gene mutations, and characterized by different pathogenic mechanisms, oxidative stress seems to be a relevant factor in the pathogenesis of SCA1 disease. Notably, mitochondrial dysfunction likely contributes to the development of several other neurodegenerative disorders, such as Huntington's disease and amyotrophic lateral sclerosis (DiMauro, Hirano, \& Schon, 2006). In addition, previous studies showed that other triplet-expanded proteins might impair mitochondrial biogenesis leading to neurodegeneration (Cui et al., 2006).

Our data suggest that the mtDNA-dependent COX defect in PC from SCA1 mice is rather specific. Since we found no differences in the density, distribution, and morphology of the mitochondrial network at PC level, we hypothesize the existence of factor(s) involved only in mtDNA homeostasis whose expression or activity is impaired by mutant ataxin in PC.

A wide variety of therapies for SCA1 is being investigated to preserve PCs and therefore delay disease onset and slow its progression. Mouse models, particularly the $154 \mathrm{Q} / 2 \mathrm{Q}$ mouse, provide a valuable model of the disease. While stem cell therapies have currently reached the clinical trial stage, recent progress in gene and protein delivery appear to be equally promising for SCA1 treatment (Wagner, O'Connor, Donsante, \& Boulis, 2016). The mitochondria-targeted antioxidant molecule MitoQ also ameliorated mitochondrial morphology and restored the activities of the electron transport chain complexes in SCA1 mice (Stucki et al., 2016).

Overall, our data show that the mtDNA content parallels the oxidative dysfunction demonstrated by histochemical evidence of COX deficiency in SCA1 mice cerebella and that the mitochondrial oxidative defect occurs as early as 8 weeks of age.

\section{ACKNOWLEDGMENTS}

This work was supported by Fondazione Cariplo Grant 2014-1010 to Dario Ronchi and Associazione Amici del Centro Dino FerrariUniversity of Milan. The authors are very grateful to Dr. Massimo 
Pandolfo from Université Libre de Bruxelles (Belgium), for kindly supplying SCA1 mice.

\section{CONFLICT OF INTEREST}

The authors have no conflict of interests to declare.

\section{AUTHOR CONTRIBUTIONS}

All authors had full access to all the data in the study and take responsibility for the integrity of the data and the accuracy of the data analysis. Conceptualization, M.R., V.L., D.R., S.C., G.P.C., M.M., and M.Sc.; Investigation, M.R., V.L., D.R., G.F., A.B., F.F., S.B., and M. M.; Writing - Original Draft, M.R., D.R., G.P.C., M.M., and M.Sc; SM performed statistical analysis. All authors read and approved the final manuscript.

\section{ORCID}

Michela Ripolone (DD http://orcid.org/0000-0001-9293-6823

\section{REFERENCES}

Allen, S. P., Duffy, L. M., Shaw, P. J., \& Grierson, A. J. (2015). Altered age-related changes in bioenergetic properties and mitochondrial morphology in fibroblasts from sporadic amyotrophic lateral sclerosis patients. Neurobiology of Aging, 36(10), 2893-2903.

Barca, E., Musumeci, O., Montagnese, F., Marino, S., Granata, F., Nunnari, D., ... Toscano, A. (2016). Cerebellar ataxia and severe muscle CoQ10 deficiency in a patient with a novel mutation in ADCK3. Clinical Genetics, 90(2), 156-160.

Bonilla, E., Schotland, D. L., DiMauro, S., \& Aldover, B. (1975). Electron cytochemistry of cristalline inclusions in human skeletal muscle mitochondria. Journal of Ultrastructure Research, 51(3), 404-408.

Bresolin, N., Moggio, M., Bet, L., Gallanti, A., Prelle, A., Nobile-Orazio, E., ... Scarlato, G. (1987). Progressive cytochrome c oxidase deficiency in a case of Kearns-Sayre syndrome: Morphological, immunological and biochemical studies in muscle biopsies and autopsy tissues. Annals of Neurology, 21(6), 564-572.

Burright, E. N., Clark, H. B., Servadio, A., Matilla, T., Feddersen, R. M., Yunis, W. S., ... Orr, H. T. (1995). SCA1 transgenic mice: A model for neurodegeneration caused by an expanded CAG trinucleotide repeat. Cell, 82(6), 937-948.

Chen, H., \& Chan, D. C. (2009). Mitochondrial dynamics-fusion, fission, movement, and mitophagy-in neurodegenerative diseases. Human Molecular Genetics, 18(R2), R169-R176.

Clark, H. B., Burright, E. N., Yunis, W. S., Larson, S., Wilcox, C., Hartman, B., ... Orr, H. T. (1997). Purkinje cell expression of mutant allele of SCA1 in transgenic mice leads to disparate effects on motor behaviours, followed by a progressive cerebellar dysfunction and histological alterations. The Journal of Neuroscience, 17(19), 7385-7395.

Cui, L., Jeong, H., Borovecki, F., Parkhurst, C. N., Tanese, N., \& Krainc, D. (2006). Transcriptional repression of PGC- $1 \alpha$ by mutant huntingtin leads to mitochondrial dysfunction and neurodegeneration. Cell, 127(1), 59-69.

Di Bella, D., Lazzaro, F., Brusco, A., Plumari, M., Battaglia, G., Pastore, A. ... Taroni, F. (2010). Mutations in the mitochondrial protease gene AFG3L2 cause dominant hereditary ataxia SCA28. Nature Genetics, 42(4), 313-321.

DiMauro, S., Hirano, M., \& Schon, E. A. (2006). Mitochondrial medicine (Phoenix Press ed.). New Haven, CT: Phoenix Press.
DiMauro, S., \& Schon, E. A. (2008). Mitochondrial disorders in the nervous system. Annual Review of Neuroscience, 31, 91-123.

Duenas, A. M., Goold, R., \& Giunti, P. (2006). Molecular pathogenesis of spinocerebellar ataxias. Brain: A Journal of Neurology, 129(Pt 6), 1357-1370.

Edwards, R. A. (2007). Laser capture microdissection of mammalian tissue. Journal of Visualized Experiments, 8, 309.

Fernandez-Funez, P., Nino-Rosales, M. L., de Gouyon, B., She, W. C., Luchak, J. M., Martinez, P., ... Botas, J. (2000). Identification of genes that modify ataxin-1-induced neurodegeneration. Nature, 408(6808), 101-106.

Gironi, M., Lamperti, C., Nemni, R., Moggio, M., Comi, G., Guerini, F. R., ... DiMauro, S. (2004). Late-onset cerebellar ataxia with hypogonadism and muscle coenzyme Q10 deficiency. Neurology, 62(5), 818820.

Horvath, R., Czermin, B., Gulati, S., Demuth, S., Houge, G., Pyle, A., ... Chinnery, P. F. (2012). Adult-onset cerebellar ataxia due to mutations in CABC1/ADCK3. Journal of Neurology, Neurosurgery, and Psychiatry, 83(2), 174-178.

Hosp, F., Vossfeldt, H., Heinig, M., Vasiljevic, D., Arumughan, A., Wyler, E., Genetic and Environmental Risk for Alzheimer's Disease GERAD1 Consortium, ... Selbach, M. (2015). Quantitative interaction proteomics of neurodegenerative disease proteins. Cell Reports, 11(7), 1134-1146.

Ito, H., Fujita, K., Tagawa, K., Chen, X., Homma, H., Sasabe, T., ... Okazawa, H. (2015). HMGB1 facilitates repair of mitochondrial DNA damage and extends the lifespan of mutant ataxin-1 knock-in mice. EMBO Molecular Medicine, 7(1), 78-101.

Kann, O., \& Kovács, R. (2007). Mitochondria and neuronal activity. American Journal of Physiology. Cell Physiology, 292(2), C641-C657.

Maltecca, F., Magnoni, R., Cerri, F., Cox, G. A., Quattrini, A., \& Casari, G. (2009). Haploinsufficiency of AFG3L2, the gene responsible for spinocerebellar ataxia type 28 , causes mitochondria-mediated Purkinje cell dark degeneration. The Journal of Neuroscience, 29(29), 9244-9254.

Matoba, R., Kato, K., Saito, S., Kurooka, C., Maruyama, C., Sakakibara, Y. \& Matsubara, K. (2000). Gene expression in mouse cerebellum during its development. Gene, 241(1), 125-131.

Nikali, K., Suomalainen, A., Saharinen, J., Kuokkanen, M., Spelbrink, J. N., Lönnqvist, T., \& Peltonen, L. (2005). Infantile onset spinocerebellar ataxia is caused by recessive mutations in mitochondrial proteins Twinkle and Twinky. Human Molecular Genetics, 14(20), 2981-2990.

Oberdick, J., Smeyne, R. J., Mann, J. R., Zackson, S., \& Morgan, J. I. (1990). A promoter that drives transgene expression in cerebellar Purkinje and retinal bipolar cells. Science (New York, N.Y.), 248(4952), 223-226.

Orr, H. T. (2012). SCA1-phosphorylation, a regulator of Ataxin-1 function and pathogenesis. Progress in Neurobiology, 99(3), 179-185.

Paulson, H. L., Bonini, N. M., \& Roth, K. A. (2000). Polyglutamine disease and neuronal cell death. Proceedings of the National Academy of Sciences of the United States of America, 97(24), 12957-12958.

Peverelli, L., Testolin, S., Villa, L., D’Amico, A., Petrini, S., Favero, C., .. Moggio, M. (2015). Histologic muscular history in steroid-treated and untreated patients with Duchenne dystrophy. Neurology, 85(21), 1886-1893.

Ripolone, M., Ronchi, D., Violano, R., Vallejo, D., Fagiolari, G., Barca, E., ... Moggio, M. (2015). Impaired muscle mitochondrial biogenesis and myogenesis in spinal muscular atrophy. JAMA Neurology, 72(6), 666675. 
Sánchez, I., Balagué, E., \& Matilla-Dueñas, A. (2016). Ataxin-1 regulates the cerebellar bioenergetics proteome through the GSK3 $\beta-m$ TOR pathway which is altered in spinocerebellar ataxia type 1 (SCA1). Human Molecular Genetics, 25(18), 4021-4040.

Sciacco, M., \& Bonilla, E. (1996). Cytochemistry and immunocytochemistry of mitochondria in tissue sections. Methods in Enzymology, 264, 509-521.

Sciacco, M., Fagiolari, G., Lamperti, C., Messina, S., Bazzi, P., Napoli, L., ... Moggio, M. (2001). Lack of apoptosis in mitochondrial encephalomyopathies. Neurology, 56(8), 1070-1074.

Shahbazian, M. D., Orr, H. T., \& Zoghbi, H. Y. (2001). Reduction of Purkinje cell pathology in SCA1 transgenic mice by p53 deletion. Neurobiology of Disease, 8(6), 974-981.

Spinazzola, A., Viscomi, C., Fernandez-Vizarra, E., Carrara, F., D'Adamo, P., Calvo, S., ... Zeviani, M. (2006). MPV17 encodes an inner mitochondrial membrane protein and is mutated in infantile hepatic mitochondrial DNA depletion. Nature Genetics, 38(5), 570-575.

Stucki, D. M., Ruegsegger, C., Steiner, S., Radecke, J., Murphy, M. P., Zuber, B., \& Saxena, S. (2016). Mitochondrial impairments contribute to spinocerebellar ataxia type 1 progression and can be ameliorated by the mitochondria-targeted antioxidant MitoQ. Free Radical Biology \& Medicine, 97, 427-440.

Vandaele, S., Nordquist, D. T., Feddersen, R. M., Tretjakoff, I., Peterson, A. C., \& Orr, H. T. (1991). Purkinje cell protein 2 regulatory regions and transgene expression in cerebellar compartments. Genes Development, 5(7), 1136-1148.

Wagner, J. L., O'Connor, D. M., Donsante, A., \& Boulis, N. M. (2016). Gene, stem cell, and alternative therapies for SCA 1. Frontiers in Molecular Neuroscience, 9, 67.
Watase, K., Weeber, E. J., Xu, B., Antalffy, B., Yuva-Paylor, L., Hashimoto, K., ... Zoghbi, H. Y. (2002). A long CAG repeat in the mouse Sca1 locus replicates SCA1 features and reveals the impact of protein solubility on selective neurodegeneration. Neuron, 34(6), 905-919.

Winterthun, S., Ferrari, G., He, L., Taylor, R. W., Zeviani, M., Turnbull, D. M., ... Bindoff, L. A. (2005). Autosomal recessive mitochondrial ataxic syndrome due to mitochondrial polymerase gamma mutations. Neurology, 64(7), 1204-1208.

Zoghbi, H. Y. (1995). Analysis of the CAG repeat and gene product in spinocerebellar ataxia type1. Proceedings of the Association of American Physicians, 107(2), 231-236.

Zoghbi, H. Y., \& Orr, H. T. (1995). Spinocerebellar ataxia type 1. Seminars in Cell Biology, 6(1), 29-35.

Zoghbi, H. Y., \& Orr, H. T. (2009). Pathogenic mechanisms of apolyglutamine-mediated neurodegenerative disease, spinocerebellar ataxia type1. Journal of Biological Chemistry, 284(12), 7425-7429.

\section{SUPPORTING INFORMATION}

Additional Supporting Information may be found online in the supporting information tab for this article.

How to cite this article: Ripolone M, Lucchini V, Ronchi D, et al. Purkinje cell COX deficiency and mtDNA depletion in an animal model of spinocerebellar ataxia type 1. J Neuro Res. 2018;96: 1576-1585. https://doi.org/10.1002/jnr.24263 